\title{
Self-Localization Algorithm for a Mobile Air Cleaner
}

\author{
Young-Baek Kim, Jin-Hee Cho and Sang-Yong Rhee \\ Division of Computer Science and Engineering, Kyungnam University \\ 449 Wolyong-dong, Masan, Gyeongnam, Korea
}

\begin{abstract}
The mobile air cleaner analyzes a distribution of polluted air to purify the air fast, calculates an optimal position and moves the position so that it can remove indoor dust more quickly. At this time, the self-localization is necessary to make the mobile air cleaner move accurately to the calculated position. This paper proposed a self-localization algorithm having high accuracy without complicated calculation and implemented it by combining a ultrasonic sensor and video processing technologies, so that it is suitable for a mobile air cleaner, and as a test result, the mean error of $\pm 1 \mathrm{~cm}$ appeared between the actually measured position and the calculated position.
\end{abstract}

Key Words : Air cleaner, Self-localization, Image processing, Ultrasonic sensor

\section{Introduction}

Indoor air is polluted by various sources, such as hazardous substances coming out from building materials or wall paper, gas and smell generated during cooking, hair of pets, etc. and brings several diseases to contemporary people spending most of time indoors [1]. Recently, houses or offices using an air cleaner are increasing to solve this problem.

However, many air cleaners released until now are used in a fixed state even though there are wheels, so it takes much time to purify all polluted air because purifying process partially.

In order to solve this problem, the air cleaner analyzes a distribution of polluted air through a sensor network to purify polluted air quickly, calculates an optimal position, moves to the position and purifies air in the precedent research[2]. The mobile air cleaner needs ability to move, but it should recognize its current position accurately. Then, it can move to a position that can purify polluted air. Accordingly, this paper aims to propose a self-localization algorithm suitable for the mobile air cleaner suggested in the precedent research[2] and implement it.

As a technique for self-localization, a method using a ultrasonic senor and a method using video obtained by using a camera are mainly used, a SLAM algorithm using only a ultrasonic senor is at MIT in USA[3]. The self-localization error of this algorithm is around $40 \mathrm{~cm}$ in case of traveling of $100 \mathrm{~m}$. Although its implement was possible with only a lowpriced ultrasonic sensor, it has a limitation that can be applied to only an environment composed of static and a straight line due to an inaccuracy.

CMU proposed an algorithm that performs self-localization,

Manuscript received Aug..17. 2009 ; revised Nov. 30. 2009.

This work is supported by Kyungnam University Foundation

Grant 2009. map formation and self-driving at the same by using only 16 ultrasonic sensors[4]. This algorithm can be applied in a corridor environment formed in a straight line, and the shape itself is being used as a marker. But this algorithm can be applied to only an environment composed of static and a straight line, and has a limitation that the total algorithm can fail due to wrong moment of a sensor input.

A tough self-localization algorithm using a probabilistic calculation method was developed at other laboratory of CMU[5]. This algorithm roughly operates an amid environment with many people, and was also actually used as selflocalization algorithm of a guide robot in the Smithonian Museum of USA. However, there are two shortcomings that we should previously know an accurate map to realize this algorithm and a laser sensor more than one should be used.

A research team of KAIST has successfully developed a selflocalization algorithm recognizing a relative marked position by sensing an artificial marker through camera image processing technologies[6]. However, this method has limitation that an artificial marker should be used, and that a position error increases as the distance between a robot and a marker gets farther, and it cannot be used in a dark environment.

However, this paper aims to solve a problem of inaccuracy easily that the self-localization technology using only the existing ultrasonic sensor and a problem that much calculation amount is generated and it is difficult to operate at a dark place by uniting these two technologies by using only image processing. Namely, the inaccuracy of a method using an ultrasonic sensor is compensated with a simple form of marker

and image processing technique using an image with low resolution, so it was made to have high accuracy with comparatively less computation. And if this simple marker is used, it is not necessary to recognize a specific color pattern, so it is possible to operate in the night if the marker is made to be exposed to light.

The paper finds out an operating principle of a mobile air cleaner in the chapter 2, describes a self-localization algorithm 
suggested in the chapter 3 , explains results of experiments in the chapter 4 and makes a conclusion in the chapter 5 .

\section{Mobile Robot for Fast Air Purification}

The operating principle of a mobile air cleaner suggested in the precedent research[2] is same as the block diagram of Fig. 2.1, and it obtains information of dust using a sensor, collects it through a sensor network and measures a distribution level.

And the optimal position that an air cleaner should be located is calculated on the basis of the measured distribution level and its current position is recognized. Then, if a path for an optimal position is decided, it moves to a calculated position by sending a control signal to its motor, and removes polluted air faster by changing its wind direction to a direction dust exists.

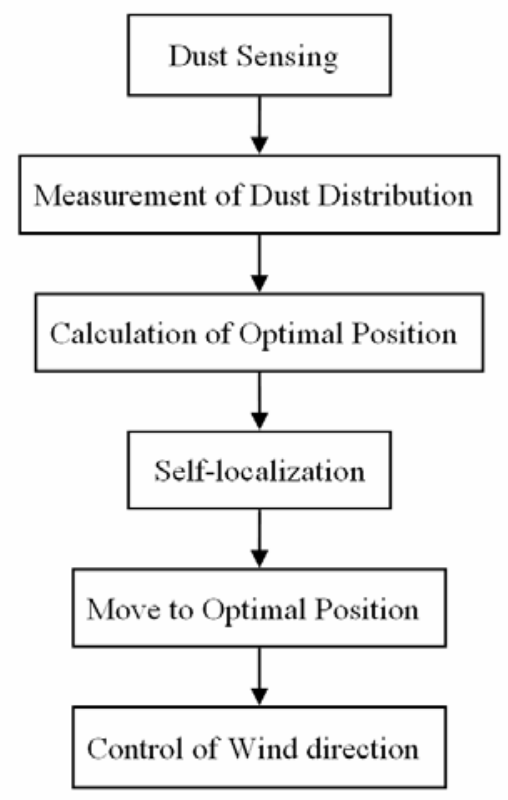

Fig. 2.1 Block Diagram

\subsection{Measurement of Dust Distribution}

The dust sensor nodes that ZigBee communication is possible are installed in a fixed interval in the room to find out the distribution of dust, that is, contaminants spread in the indoor air. If these sensor nodes detect dust in its position, they send detected dust information and its ID to a sensor coordinator. The sensor coordinator is a base station receiving sensor node's ID and dust information that the sensor nodes send distribution at several places Then, it understands current distribution of dust spread in the room by mapping sensor node's ID and coordinates that dust is detected.

\subsection{Calculation of Optimal Position}

Even though the dust distribution is same in the room, its wind direction control changes according to a position of an air cleaner. In other words, it means that the angle for sending wind should be adjusted according to dust distribution. Fig. 2.2 shows wind direction control according to dust distribution and a position of an air cleaner, and it indicates a circle as a dust distribution and a square as an air cleaner.
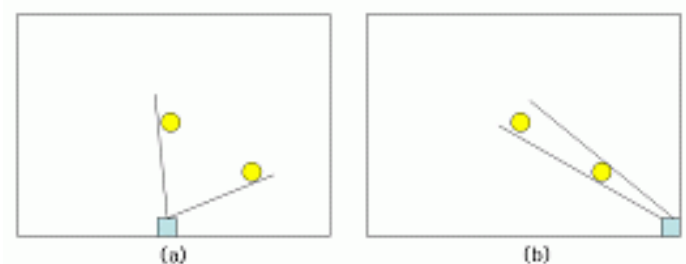

Fig. 2.2 Wind direction control according to dust distribution and a position of an air cleaner

Here, according to the dust distribution in (a) and (b) of Fig. 2.2, an important point is that the wind direction adjustment angle of (b) in Fig. 2.2 is much narrow according to a position of an air cleaner. In case of (a) of Fig. 2.2, this means that as it send wind to a portion that dust is not distributed while moving wind direction to each dust position, it takes longer to remove dust. Accordingly, if there is an air cleaner at a position like (a) of Fig. 2.2, dust can be removed faster by moving the air cleaner like (b) in Fig. 2.2.

In order to move an air cleaner to an optimal position for dust removal, we should first know where the optimal position is. The optimal position for dust removal can be found by the following method.

step 1. Initialization of Air Cleaner's Position

step 2. Calculation of Wind Direction Control Angle and Distance according to Dust Distribution

step 3. Storage at a position that angle $\times$ distance is minimum in comparison with previous data.

step 4. Movement of Air Cleaner's Position

step 5. Repetition of step 2 4 until an air cleaner reaches an end position

Of course, as this is calculated with software, the time for its calculation can be almost neglected.

\subsection{Simulation}

We simulated the proposed mobile air cleaner and evaluated the performance before constructing the complete system. Fig 2.3 is the first screen of the simulation program.

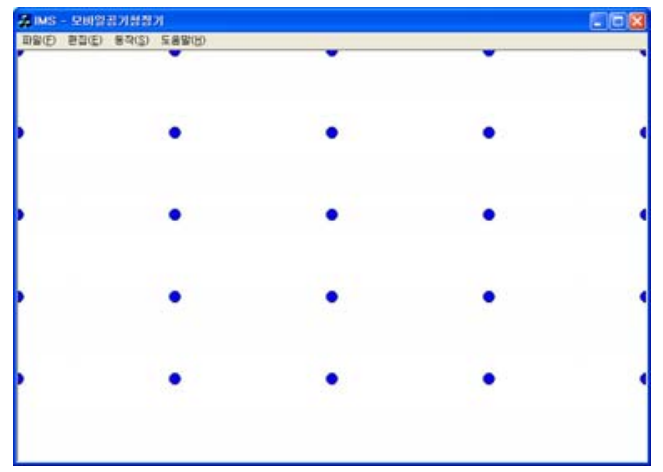

Fig. 2.3 The first of Simulation Program 
The simulation area was assumed to indoor space. And we also assumed that the dots were dust sensor nodes and the mobile air cleaner could move along the wall that was described as the bottom line. And we simulated the two cases, one was dust sensors are 4 by 4 and another was 5 by 5 as shown Fig 2.3.

We used the evaluation measure as a distance between the air cleaner and the sensor that detected dust products blow angle range for all of the case when dust happened. We compared and evaluated three cases of positions. One is that fixed air cleaner on the center of the wall. Second case is that air cleaner was fixed on the corner of the room. The last case was a mobile air cleaner. A criterion is first case, the fixed air cleaner on the center of the wall. Table 1 is the result in this case that 4 by 4 sensor nodes were installed.

Table 1 . Simulation on $4 \times 4$ sensor nodes

\begin{tabular}{|c|c|c|c|}
\hline & Center & Corner & $\begin{array}{c}\text { The proposed } \\
\text { method }\end{array}$ \\
\hline $\begin{array}{c}\text { Relative energy } \\
\text { to clean }\end{array}$ & 100 & 85 & 57 \\
\hline
\end{tabular}

As shown in table 1, the proposed method is better than cases that the air cleaners are fixed by around $43 \%$.

Table 2 is the result of the case that 5 by 5 sensor nodes were installed

Table 2 . Simulation on $5 \times 5$ sensor nodes

\begin{tabular}{|c|c|c|c|}
\hline & Center & Corner & $\begin{array}{c}\text { The proposed } \\
\text { method }\end{array}$ \\
\hline $\begin{array}{c}\text { Relative energy } \\
\text { to clean }\end{array}$ & 100 & 83 & 51 \\
\hline
\end{tabular}

As shown in table 2, the proposed method is better than cases that the air cleaners are fixed by around $49 \%$. If number of sensor nodes is increased, we could easily find the dust and know the accurate dust distribution and clean the dust more effectively. But purchasing cost and operating cost would be increased.

\section{Self-localization Algorithm}

The ultrasonic sensor has merits that its price is cheap and its calculation for self-localization takes short. Accordingly, the method recognizing its position through a triangulation survey method after an ultrasonic sensor was mounted on a sensor node for measuring dust distribution could become the most economical method. However, if a plural of ultrasonic sensors respectively shoot ultrasonic wave, it becomes difficult that a listener recognizes it. If ultrasonic wave was linearly transmitted and recognized directly, there is no problem, but when the ultrasonic wave bounded by a wall or a hindrance in a transmitting process, if could be wrongly recognized as ultrasonic wave transmitted by other Beacon.
It is not easy to correct all these things, and more computations are necessary in order to fix it and there is no guarantee that it is accurately corrected.

In order to solve this problem, this paper proposes a selflocalization algorithm with an ultrasonic sensor and a camera image. The biggest limitation of a self-localization method using a camera image is to need an image with high resolution for recognition of an accurate position and to demand computations that a processor mounted on a mobile air cleaner are difficult to handle. Namely, in order to recognize a position by using a marker, after a calibration process is carried out and ID was given to each marker, it should be recognized, so an image with high resolution is necessary and many computations are required due to it.

However, this paper aims to detect a marker even in an image with low resolution by making a marker with simple form that can be recognized easily, obtains ID of a marker difficult to indicate with a simple marker and the distance between markers in an air cleaner by using one ultrasonic sensor. Additionally, thesis aims to enhance accuracy and reduce calculation necessarily for image processing at the same time by simplifying the self-localization algorithm and reducing potential errors of the algorithm.

\subsection{Viewpoint}

The self-localization algorithm proposed in this paper supposes that the height from the floor to the ceiling is all the same. After the $h$ value was set as the height of an air cleaner subtracted from the height from the floor to the ceiling of Fig. 3.1, the $r$ value of Fig. 3.1 is obtained by using an ultrasonic sensor. Then the $d$ value is obtained with the $r$ value and the previously measured distance $h$ which is from a mobile robot to the ceiling by using Pythagorean Theorem. The equation of a circle with a radius of $d$ that the Beacon position becomes the center as shown in Fig. 3.2 can be obtained.

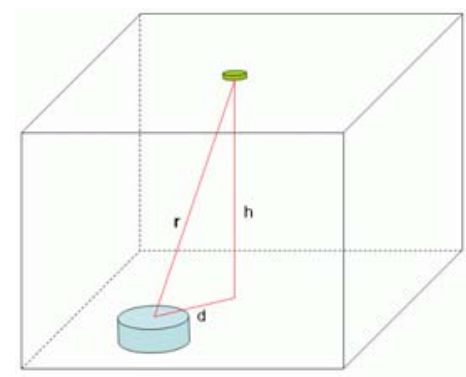

Fig. 3.1 Calculation of distance using an ultrasonic sensor 


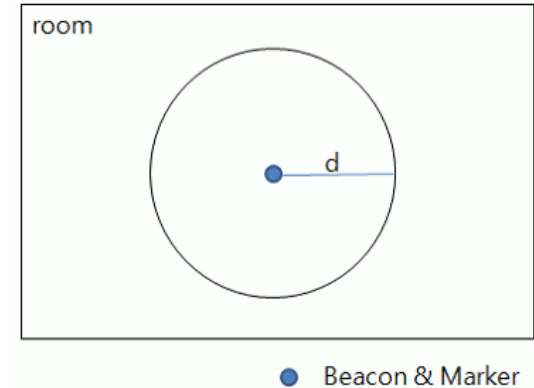

Fig. 3.2 Expectable position of a Mobile Robot

Once the process has been done, we can know a position of a mobile robot is located somewhere inside a circle of Fig. 3.2. However, because we don't know where the mobile robot is exactly located inside a circle, this maker can be recognized in an image obtained through a camera by attaching a marker to a position of Beacon like Fig. 3.3 to solve this. After it is found out how much the marker is spread in a $\mathrm{x}$ or $\mathrm{y}$ axis direction from the center of an image by using a position of recognized marker, the $d$ value obtained by using an ultrasonic sensor is matched with the distance from the center of the image to the marker. Then its own position could be recognized by obtaining relative coordinates about how much the mobile robot is spread from the position of the marker and the Beacon in $\mathrm{a} x$ or $y$ axis direction.

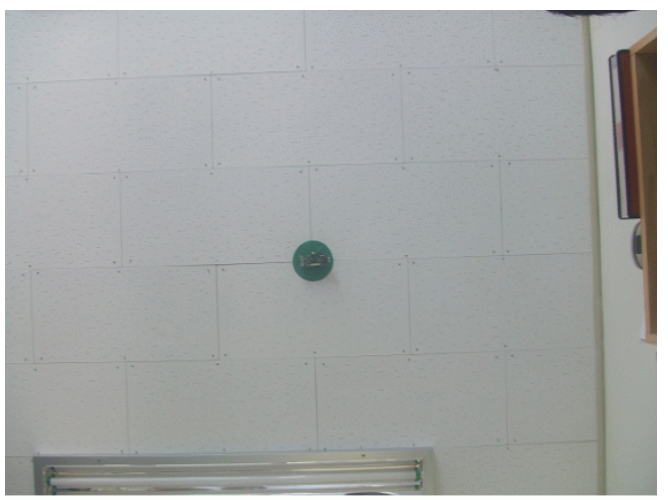

Fig. 3.3 Marker attached to the ceiling

\subsection{Calculation of Distance between Marker and Mobile Robot using Ultrasonic Sensor}

The basic distance calculation using an ultrasonic sensor is the same as in Fig. 3.4.

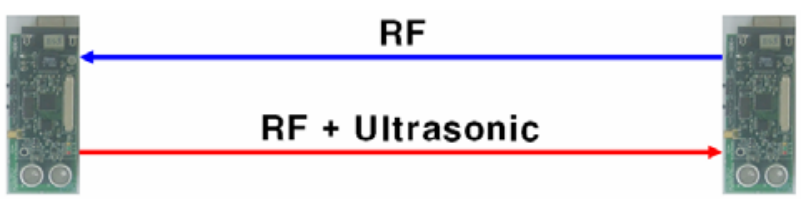

Fig. 3.4 Operating Method of an Ultrasonic Sensor

First, if the listener sends a RF signal to Beacon, the Beacon receiving this signal sends the $\mathrm{RF}$ signal and an ultrasonic signal at the same time. Then the listener receives two signals and calculates the distance to Beacon by using the time difference of two signals. The velocity formula of ultrasonic wave should be first known to calculate the distance like this, and the formula is same as the equation (1).

$$
v(\mathrm{~m} / \mathrm{s})=331.5+0.60714 T
$$

Here, $v$ is velocity, and $T$ is temperature. If the temperature is supposed to be $20^{\circ}$, the velocity of ultrasonic wave is $343 \mathrm{~m} / \mathrm{s}$. Accordingly, the distance between the Beacon and the listener can be obtained by multiplying times(sec) calculated with a timer by 343 . However, if the distance information received from the listener is analyzed, a problem that the listener cannot receive ultrasonic wave sent from the Beacon often occurred in a process calculating the distance. Thus in this paper the median value of measured data that received 5 times was used as its actual distance data to filter these error data out.

\subsection{Marker Detection and Self-localization inside an Image}

We made the marker for recognizing locations as in a circle, so it can have same portion. Even though it takes an image in different position, and to distinguish the regions of the background and the marker for value of RGB color-model, green which has lowest distribution in the background is chosen for the marker's color.

To detect the marker, a color image like Figure 3.3 is acquired. And the equation (2) represents the process to normalization on RGB color-valued of obtained image.

$$
r=\frac{R}{R+G+B}, g=\frac{G}{R+G+B}
$$

Because the relation $r+g+b=1$ exists, $b$ ratio is not used. After normalizing as like the above, average value and covariance is calculated for the marker region by using formula (3).

$$
\begin{aligned}
& \bar{r}=\frac{1}{N} \sum_{i=1}^{N} r_{i}, \bar{g}=\frac{1}{N} \sum_{i=1}^{N} g_{i} \\
& \sum=\left[\begin{array}{l}
\sigma_{r r} \sigma_{r g} \\
\sigma_{g r} \sigma_{g g}
\end{array}\right]
\end{aligned}
$$

By using average value and covariance of formula (3), we got an intensity image of marker candidate region as Figure 3.5. If pixels have high relation with the color of the marker, they have higher value between 0 and 255 . 


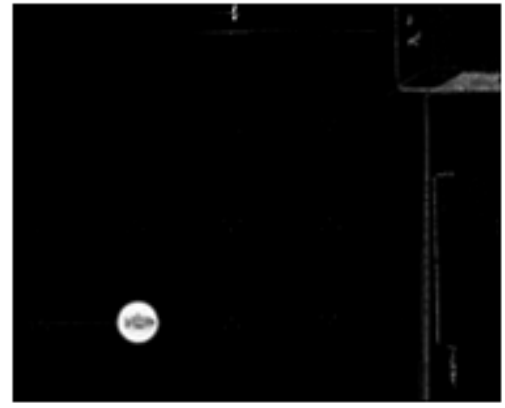

Fig. 3.5 Intensity Image of Marker Candidate Region

In Figure 3.5, there are some not black color regions however they are not marker region. That's why there are some regions that have similar RGB value of the marker. But because those regions have lower correlation, binarization process using a proper threshold value can give us a good result like Figure 3.7 that shows only in marker region.

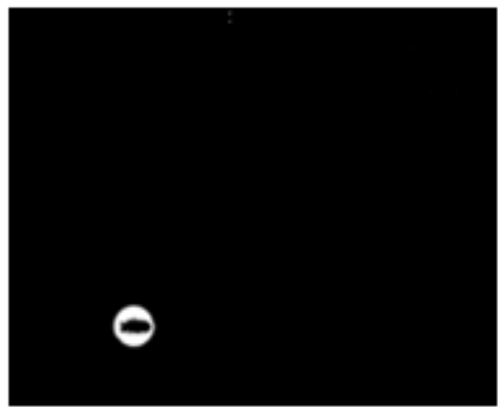

Fig. 3.6 Marker Detection using Binarization

We can find the center position of the maker by blob analysis on extracted marker region. Blob analysis allows you to identify connected regions of pixels within an image, then calculate selected features of those regions. The regions are commonly known as blobs.

Blobs are areas of touching pixels that are in the same logical pixel state. This pixel state is called the foreground state, while the alternate state is called the background state. Typically, the background has the value zero and the foreground is everything else.

The marker's coordinates by analyzing blob can be figure out by the relative state of the mobile air cleaner on the marker's location using calibration of $d$ value shown in the Figure 3.1. And the calculated relative position like this, we can get the real position of the mobile air cleaner after adding the offset of the real marker position.

\section{Experiment \& Result Analysis}

In this paper, the self-localization was carried out by attaching a color marker capable of recognizing through a camera and a ultrasonic sensor(beacon) to the ceiling and installing a listener and a camera to a computer corresponding with a mobile air cleaner. The proposed algorithm was experimented in a Pentium PC by using an ultrasonic sensor of Hybus Co. and Webcam of Logitec Co., and the experiment's equipments were placed at a position that is separated by $30 \mathrm{~cm}$, $50 \mathrm{~cm}$ and $1 \mathrm{~m}$ from a position of a marker and an ultrasonic sensor in the experiment and its error was measured. Its experiment's results are shown in Table 3.

Table 3

\begin{tabular}{|c|c|c|}
\hline \multicolumn{2}{|c|}{ Experimental Result } & Unit: cm \\
\hline Position & Maximum Error & Mean Error \\
\hline 30 & 6.13 & 1.77 \\
\hline 50 & 3.97 & 0.85 \\
\hline 100 & 2.21 & 0.6 \\
\hline
\end{tabular}

If the experiment's results of Table 1 are noted, it can be seen as the position is closer, the error is increased, and as the position gets farther, the error is reduced. This is caused by an error of distance information coming from an ultrasonic sensor. As the distance information coming from an ultrasonic sensor corresponds to a hypotenuse of a triangle in Pythagorean theorem, the error of this hypotenuse has bigger influence on what seeks a distance value of the base line, as the distance is closer. And when it was observed overall, the mean error range was around $\pm 1 \mathrm{~cm}$, so it showed a good result in operating a mobile air cleaner.

\section{Conclusion}

The mobile air cleaner was proposed to purify polluted air faster than the existing fixed air cleaner, and this mobile air cleaner should accurately move to a position that can remove polluted air fast and thus needs self-localization. Accordingly, this paper proposed a self-localization algorithm suitable for a mobile air cleaner and implemented it.

For self-localization of a mobile air cleaner, the two dimentional distance was simply sought by receiving distance information from an ultrasonic sensor and using Pythagorean theorem, and a method recognizing a position of an actual mobile robot by using distance information obtained previously after recognizing a marker and seeking screen coordinates on the screen through vision technology. So it showed selflocalization performance have a mean error range of $\pm 1 \mathrm{~cm}$.

The proposed method solved a difficult problem, which results from what only an ultrasonic sensor or vision technology was used, by using mutual complementing elements of two technologies and uniting it. It is expected that as the complicated calculation or filtering is not necessary due to this method, it could be utilized for a mobile air cleaner with low hardware specification.

\section{References}

[1] “Keeper of indoor air- Air Cleaner,” House full of Happiness, no.7, pp. 11, 2009. 
[2] Sang-Yong Rhee, Young-Baek Kim and Jin-Hee Cho, "A Mobile Air Cleaner to Clean Dust Rapidly," International Symposium on Robotics, pp. 948-951, 2008.

[3] J. D. Tardos, J. Neira, P. M. Newman and J. J. Leonard, "Robust mapping and localization in indoor environments using sonar data," International Journal of Robotics Research, vol. 21, no. 4, pp. 311-330, 2002.

[4] H. Choset and K. Nagatani, "Topological SLAM toward exact localization without explicit localization," IEEE Trans. on Robotics and Automation, vol. 17, no.2, pp. 125-137, 2001.

[5] S. Thrun, D. Fox, W. Burgard and F. Dellaert, "Robust monte carlo localization for mobile robots," Artificial Intelligence, vol. 128, pp. 99-141, 2001.

[6] G. Jang, S. H. Lee and I. Kweon, "Color landma based selflocalization for indoor mobile robots," Proc. of IEEE Int. Conf. on Robotics and Automation, pp. 1037-1042, 2002.

[7] P. Newman, J. Leonard, J. D. Tardos and J. Neira, "Explore and return : experimental validation of real-time concurrent mapping and localization," Proc. of IEEE Int. Conf. on Robotics and Automation, pp. 1802-1809, 2002.

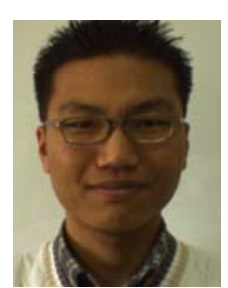

\section{Young-Baek Kim}

2005 : Graduation from Division of Computer Science and Engineering, Kyungnam University. 2007: Graduate School of Division of Computer Science and Engineering, Kyungnam University (MS).

2007 Present: Graduate School of Division of Computer Science and Engineering, Kyungnam University ( $\mathrm{PhD}$ Course) Interest Field : Image Processing, Context Awareness, Augmented Reality

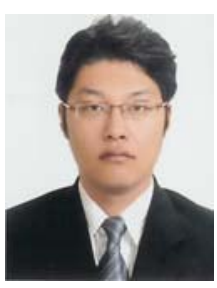

Jin-Hee Cho

2007 : Graduation from Division of Computer Science and Engineering, Kyungnam University. 2007 Present : Graduate School of Division of Computer Science and Engineering, Kyungnam University (MS course)

Interest Field : Senor Network

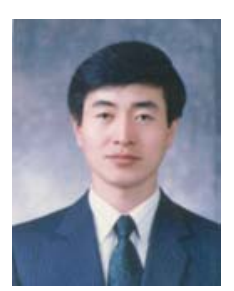

\section{Sang-Yong Rhee}

1982 : Graduation from Department of Industrial Engineering, Korea University 1984 : Department of Industrial Engineering, Graduate School, Korea University (MS)

1992: Department of Industrial Engineering, Graduate School, Postech (PhD)

1992 Present : Professor, Division of Computer Science and Engineering, Kyungnam University

Interest Field: Computer Vision, Neuro Fuzzy, Intelligent Robot, Biometrics 